\title{
El objeto olvidado de la sociología
}

\author{
Francisco Parra Luna \\ Universidad C omplutense de M adrid. Facultad de C iencias Políticas y Sociología \\ 28023 Pozuelo de Alarcón (M adrid). Spain \\ parralun@lix.intercom.es
}

\section{Resumen}

La sociología podría haber olvidado el objeto para el cual comienza estableciéndose como ciencia. A partir del homocentrismo tradicional de la sociología, se conviene en admitir que es la persona humana (la «vieja medida de todas las cosas»), con su complejo vital de necesidades e intereses, significaciones y deseos, expectativas y cálculos, el centro de interés insoslayable de todo estudio sobre lo social. Sin embargo, formalismos como los de Simmel, unidos a la eclosión de las numerosas especialidades a que da origen el acelerado desarrollo institucional de la ciencia y el más que probable mimetismo sociológico por seguirla, han ido minando subrepticiamente el fin primigenio de la sociología (hasta llegar, por ejemplo, a la «sociedad sin hombres» de Luhmann), que no fue otro que el descubrimiento de los principios más estructurantes que determinaban o condicionaban el funcionamiento de la vida social con el fin exclusivo de la mejora de ésta última; lo que es tanto como decir, para ben eficio de las personas que la formaban. En este artículo, que enfatiza el falso dilema cualitativo-cuantitativo como perversión del lenguaje sociológico, se pretende recordar que la sociedad no podría ser sólo un compuesto de formas abstractas, sino sobre todo un conjunto interactivo y consciente de personas con necesidades concretas, las cuales viven precisamente en sociedad sólo y exclusivamente para mejor satisfacerlas. Y que la sociología es, o debería ser, ese conocimiento acumulado sobre los fenómenos sociales que coadyuve hacia la mayor satisfacción de estas necesidades.

Palabras clave: necesidad, fines, medios, eficiencia, transformación, axiología.

\begin{abstract}
The forgotten object of Sociology
Sociology seems to have forgotten the objet for which it was initially created as a science. From the early ethnocentric stream of thought in the founding fathers, human concerns (needs; interests and meanings) wore at the heart of sociological reflection. Later on, under the influence of formalists like Simmel, and the accelerated development of positive natural sciences with their small and numerous specialities, the initial objet of Sociology was increasingly substituted by some secondary aspects of society f.i. «communications» (until reaching Luhmann's «Society without men»), which are only means used by people for achieving their goals. Thus, Sociology seems to forget progressively to study the concept of goal-setting (the systems of values persued/achieved) for which society (or any type of social organization) was created, since this is the purpose justifying the existence of society. In this paper, which emphasises the spurious qualitative-quantitative
\end{abstract}


dilemma as a perversion of sociological language, I try to remain that society is not composed solely of abstract forms, but mainly of human individuals who live together because, in this way, they satisfy their mutual needs better. Sociology should or could then be the science which offers its cumulated knowledge on social phenomenon to cater for the satisfaction of these needs

Key words: need, goals, means, performance, transformation, axiology.

\section{Sumario}

1. Breve introducción al dilema cualitativo-cuantitativo 0 de cómo su exégesis empañaría el entendimiento sociológico

2. Una técnica: el método $D$ elfos 3. ¿Sociología para quién?
4. El objetivo olvidado

el «diferencial sinérgico»

5. ¿Y la teoría sistémica?

6. ¿Una hipótesis plausible?

Bibliografía

N ada habría que objetar a que, de aquella primera sociología que nos legaron Ferguson, S. Simon, Comte y demás padres fundadores, hayamos ido saltando a las diferentes sociologías especializadas (de la salud, del trabajo, de la comunicación, etc.) cual ramas que expenden la savia del joven árbol. Sociología, aquella, muy incardinada en los problemas sociales y políticos de la época, comprometida con los problemas de las masas y centrada en la persona como elemento vital y necesario de cualquier hecho social. No hay más que repasar la relación que existió entre los llamados «niveladores» (Lilburne), los «cavadores» (W instanley) o los fisiócratas (Turgot, Q uesnay) con los nuevos científicos social es (M ontesquieu, A. Smith, Saint Simon, C ondorcet... ), durante Ios sigloS XVI y XVII en la nueva Europa ilustrada. EI homocentrismo en sociología queda bien demostrado por Lemert (1979: 1-22).

Sí habría que comenzar a objetar algo cuando se constata la amnésica y progresiva desaparición del concepto de necesidad (individual o social) en tanto que inevitable componente axial y vicario de cualquier formación social. Se trata de un deslizamiento suave hacia una abstracción crecientemente descarnada que ya toma visos preocupantes en Simmel (su énfasis en las «formas») y culmina con esa teoría til dada por al gunos de «escandal osa», como es la teoría de Luhmann, para quien la persona no existe prácticamente en su enfoque ¿sistémico? de la sociedad. La desventura teórica se concreta así cuando la sociología se despreocupa del conjunto de las necesidades de las personas que componen una organización o sistema mientras permanecen en él, esto es, se desentiende precisamente de aquello para lo cual el sistema nace o se crea. Y a objetar decisivamente, cuando dicho fenómeno amnésico se reproduce en las Ilamadas sociologías de la organización, de la empresa, o de los sistemas sociales en tanto que unidades globales, por no hablar de la «sociología de la política», centrada/virada casi exclusivamente hacia los estudios el ectorales 
y el comportamiento político versus el sistema empírico de valores producido en beneficio de las poblaciones.

Y si bien la sociología no podría dejar de ocuparse de al guna u otra forma de las necesidades humanas, apenas lo hace (al menos no conozco ningún caso) de una manera global, sistemática y cuantificada (científica en suma) de las diferentes unidades sociales, y ello a pesar del importante movimiento de los indicadores sociales, del «Bauer» en EE.UU . a los FOESSA en España, que de alguna manera se preocupan de representar tales unidades. Exceptuando la aportación teórica de este movimiento, el gran resto de la sociología viene oscilando entre un microcuantofrenismo crecientemente acelerado por la facilidad de los ordenadores, y una residualizante sociología parafilosófica o ensayística que entiende de todo lo que le echen. La primera por su numerología desgarbada (teóricamente roma), y la segunda por científica e inverificable (incomunicable, por tanto). Entre las dos han configurado una sociología apenas acumulativa a pesar de los encomiables esfuerzos de Berelson, Steiner y otros, con sus tempranos inventarios de proposiciones. El resultado actual es una sociología paniaguado (una «maría» solían llamarlo los estudiantes de Económicas en España) con dos características básicas: primero, alejada de las preocupaciones diarias de la gente de la calle, y segundo, sin estatus científico respetable. 0 como reconoció hace varios años un veterano sociólogo español: «iEsto ni es ciencia ni es nada!».

Intentando resumir el estado de la cuestión, podría decirse que la sociología actual, ni se centra en lo relevante, ni lo hace de manera verificable, para presumible gozo y regocijo de al gunas élites felices con este tipo de discurrir inocuo. Y ello a pesar de un cierto avance de las teorías de la complejidad, de la proliferación de los datos cuantitativos, del potencial de cálculo de los ordenadores y de sus admirables programas de cál culo rutinizados.

Considerando por tanto que el problema se centra en el lenguaje utilizado, permítaseme una especie de rodeo desenfadado por los tres apartados siguientes: 1. La controversia cualitativo-cuantitativo; 2) La descripción de una técnica (D elfos) para pasar de lo primero a lo segundo, y 3) La aplicación de ambas técnicas para mostrar que toda sociedad produce un valor añadido (lo que es su esencia) que puede ser cuantificado y que, sin embargo no es objeto de análisis ni por la sociología ni por ninguna otra ciencia. La amnesia social queda instalada (Jacoby, 1977) y el olvido del concepto motor en la sociología fundadora asumido, al parecer sin ningún tipo de trauma, por los sociólogos practicantes.

\section{Breve introducción al dilema cualitativo-cuantitativo o de cómo su exégesis empañaría el entendimiento sociológico}

Una de las divisiones a las que nos tiene acostumbrados la sociología es la tópica adscripción de los sociólogos a los modos de hacer llamados cualitati vos (utilización del lenguaje común como instrumento) y cuantitativos (utilización del lenguaje matemático y las técnicas métricas). Adscripción que conlleva 
hacia prácticas sociológicas incomunicadas y cuyos autores más parece que se miren de soslayo que contribuyan a un conocimiento común.

La polémica como se sabe es vieja. La llamada sociología empírica (bien representado por lo que W. M ills llamó «empiricismo abstracto» americano) suele exhibir la siguiente artillería: «V ds. los sociólogos críticos, los filósofos sociales, los que no medís, y ni siquiera definís operacionalmente los conceptos que utilizáis, no podéis demostrar que vuestros argumentos son ciertos, verificables o replicables; habláis de cosas importantes, cierto, pero nos proporcionáis tantas opiniones como colegas las emiten; no sois científicos, no proporcionáis cumulatividad a vuestro conocimiento, y en suma no hacéis estricta "sociología" (ciencia de la sociedad)». Es la versión popperiana de la ciencia exigi endo el certificado de falsabilidad de toda proposición científica.

Pero los «cuitados» no son precisamente mudos y suelen granear su fuego de esta guisa: «Y ustedes los formal-matemáticos, los empiristas redomados, sois precisos (a veces hasta el ridículo) pero en cuestiones irrel evantes o que tienen una significación social escondida. I gnoráis las estructuras social es 0 políticas que dominan la producción del pequeño conocimiento que presentáis; no comprendéis nada de los intereses que mueven el mundo; y solamente utilizáis los datos que precisamente reproduce la maraña de intereses sociopolíticos en juego». Es la versión más kuhniana y libre de la ciencia representada por numerosas escuelas críticas (psicoanálisis, Escuela de Francfort, neomarxismo, etc.) o derivada de posiciones fenomenológicas (hermenéutica, etnometodología, interaccionismo simbólico, relativismo cultural, etc.). D iatriba en el fondo más apodíctica que aporética, pero que todavía se mantiene viva. A lo que contribuye la mostrenca e impresentable pedagogía matemática, que se suele utilizar en las escuelas urbi et orbi.

¿C uál de estas dos posiciones tiene razón? Seguramente ambas. Lo que resulta atrayente y además necesario es intentar integrar ambos enfoques para conseguir: a) hablar de lo social-relevante; y b) de una manera verificable o replicable. Éste fue uno de los desafíos que se planteó Von Bertalanffy con su teoría general de sistemas seguido en ciencias sociales por Easton, D eutsch, Buckley, Van Gigch, Bailey y otros. En esta línea permítaseme adelantar tres observaciones:

- Primera, contraponer lo cualitativo a lo cuantitativo viene a carecer de sentido epistemológico, puesto que no parece que pueda existir nada solo cuantitativamente (se refiere a propiedades intensionales) si previamente no se distingue cualitativamente (propiedad extensional). No se puede pre cisar lo que pesa por ejemplo el bolígrafo que uso (una de sus cualidades mensurables) si no se comienza por distinguir el objeto «bolígrafo» de otros objetos. Enfrentar, como suele hacerse, ambos enfoques metodológicos resulta tan absurdo como intentar demostrar que algo pesa $X$ sin una referencia cualitativa mínima a dicho «algo». Se puede asegurar por tanto que lo cuantitativo implica forzosamente lo cualitativo, y que la operación cua litativa (de orden semántico) es previa a la operación cuantitativa (de orden 
métrico). La cualidad es anterior y más fundamental que la cantidad, ya que no pueden existir cantidades de la «nada» 0 de lo que es desconocido o no percibido.

- Segunda, si la cualidad resulta fundamental en el origen del conocimiento, la cantidad termina siendo más enriquecedora y definitoria al representar un valor añadido, un conocimiento complementario. «El bolígrafo es ligero» 0 «El bolígrafo pesa 82 gramos» son operaciones gramaticales donde los predicados «ligero» 0 «pesa 82 gramos» añaden en cualquier caso un conocimiento que se suma siempre al conocimiento inicial que teníamos del bolígrafo (su estructura y sus funciones). Al presentarse en el proceso del conocimiento en un momento posterior, el enfoque cuantitativo (ya sea adjetivado o cifrado) termina siendo forzosamente superior o complementario al cualitativo.

- Y tercera, y en consecuencia con lo dicho, conviene denunciar la generalizada falacia que supone tomar por cualitativo lo que no es más que una operación de cuantificación deficiente. Cuando se dice «Este bolígrafo es ligero» (adjetivando en lenguaje ordinario una cualidad intensional del bolígrafo), dicha proposición puede entonces contraponerse a la expresión «Este bolígrafo pesa 82 gramos» (incorporando al conocimiento que tenemos del bolígrafo como objeto diferencia una propiedad precisa y replicable). Por tanto, el lenguaje adjetivado no puede sino proporcionar una experiencia personal subjetiva, intransferible y no falsable. Se trataría, como sostiene Lachenmeyer (1971) en su crítica a la sociología como ciencia, de un lenguaje ambiguo, vago, opaco y contradictorio, inadecuado para conformar un conocimiento científico. Por el contrario, el lenguaje métrico resulta precisamente lo contrario: claro, preciso, transparente, lógico y replicable. Confundirse no deben, pues, aquellas malas cuantificaci ones que llamamos «análisis cualitativo» (Schwartz y Jacobs, 1979) con la inevitable cualificación previa o tipología distintiva entre objetos. Problema distinto es que podamos finalmente cuantificar lo social y en especial lo que vengo Ilamando «social relevante», esto es, la performance o eficiencia de las organizaciones sociales.

\section{Una técnica: el método D elfos}

¡C uantificar lo relevante! Ahí es nada. ¿Y quién dice lo que es relevante? ¿Y qué partes o dimensiones lo son? ¿Y cuánto de relevante para unos y para otros? Y lo que hoy es relevante, ¿lo fue ayer o lo seguirá siendo mañana? Recordemos de nuevo que numerosos colegas de reconocido oficio tienen bien asumido que raramente lo social significativo puede cuantificarse satisfactoriamente. Véase p.e. el magistral y conocido (aunque hoy superado) artículo de C. M oya en los años sesenta sobre su crítica a los indicadores sociales. Por no hablar de la frase que tuve ocasión de oír personalmentea P. Berger «o social no es matematizable y si se hiciera deshumanizaríamos lo social». E specie de exabrupto analgesizante que puede haber evitado a muchos el a veces terrible dolor del 
silicio matemático. Trátase además de una especie de aspirina-trampa de inspiración conservadora, ya que contribuye a evitar que se demuestren con precisión verificable las numerosas injusticias que en el mundo son. $\mathrm{N}$ egando la posibilidad de cuantificarlas, todo puede quedarse en meras opiniones, tacha bles además de subjetivas, ideologizadas e imprecisas en cuanto así interese. Q ué duda cabe que un análisis comparativo (por referirme a una discriminación capital fáctica) de los porcentajes de mujeres parlamentarias, presidentes ejecutivas de grandes empresas, directoras de periódicos o que han alcanzado el rango que «generalas» 0 de «obispas», proporcionaría resultados de escandal osa desigualdad en una sociedad que se precia de perseguir la igualdad de oportunidades. El análisis comparativo de estos datos en sociedades diferentes, sus tendencias en el tiempo (lo que permite el ajuste a rectas matemáticas y el conocimiento de los grados exactos de cambio), su puesta en relación con posibles variables explicativas (nivel de educación, estatus socioeconómico de los padres, etc.) proporcionarían sin duda un conocimiento estadístico de estos fenómenos, no sólo más explicativo y exacto, sino también, y sobre todo, más verificable, 0 al menos falsable, que es lo que hace respetable al conocimiento y permite su cumulatividad. Triste resulta constatar que muchos colegas progresistas y bien intencionados se niegan en redondo, por unos y otros motivos, a la matematización de lo social. El lenguaje, como sabemos desde W horf y Sapir hasta Giddens, no es un mero reflejo de la realidad social, sino que la estructura y la constituye inapropiada, pues, constitución social contribuiríamos a conseguir con un lenguaje inadecuado.

Sin tomar como ejemplo tan apasionantes problemas de desigualdad genérica si deseo referirme al origen del método D elfos, diseñado precisamente para pasar del lenguaje común (en temas complejos y poco medibles) al matemático y cuantificado. Es el problema que se les presenta a ciertas empresas cuando han de decidir, por ejemplo, si conviene montar una planta industrial en el país A o en el B. Sucede que una vez efectuados los correspondientes estudios de rentabilidad económica a corto plazo es preciso también evaluar la seguridad futura de las inversiones en base a aspectos tal es como la ideología política del sistema, la estabilidad gubernamental, la presión sindical, la actitud de la Administración, las expectativas futuras u otros aspectos igualmente difusos. Conjeturas ideológicas por el momento aparte, dada la dificultad de concretar y definir con precisión estas situaciones, la empresa suele enviar a un equipo de expertos a observar in situ la situación concreta en cada uno de los países. D e vuelta, celebran varias reuniones donde cada uno de los expertos presenta una percepción cuantificada de 0 a 10 traduciendo numéricamente (graduando) los aspectos mentados subjetivos, perceptivos y en definitiva interpretativos y comprensivos de cada uno de los criterios previa e igualmente consensuados. Fruto de una primera reunión entre los ejecutivos y técnicos empresariales es la formación de dos matrices básicas como las siguientes (ejemplos hipotéticos para cuatro criterios y cinco expertos), donde se aprecian las cifras proporcionadas por cada experto para cada uno de los criterios utilizados: 
Tabla 1. País A

\begin{tabular}{lrrrrll}
\hline Criterios & Técnico 1 & $\mathbf{2}$ & $\mathbf{3}$ & $\mathbf{4}$ & $\mathbf{5}$ & Promedio \\
\hline 1 Ideología política & 6 & 5 & 6 & 6 & 7 & 6 \\
2 Presencia sindical & 10 & 10 & 8 & 9 & 9 & 9 \\
3 Estabilidad Gobierno & 4 & 4 & 6 & 6 & 5 & 5 \\
4 Acutación Administración & 8 & 8 & 6 & 8 & 7 & 7,4 \\
\hline
\end{tabular}

Tabla 2. País B

\begin{tabular}{lrrrrll}
\hline Criterios & Experto $\mathbf{1}$ & $\mathbf{2}$ & $\mathbf{3}$ & $\mathbf{4}$ & $\mathbf{5}$ & Promedio \\
\hline 1 Ideología política & 6 & 4 & 6 & 5 & 4 & 5 \\
2 Presencia sindical & 9 & 8 & 9 & 10 & 9 & 9 \\
3 Estabilidad Gobierno & 2 & 1 & 2 & 3 & 2 & 2 \\
4 Actuación Administración & 2 & 2 & 2 & 1 & 1 & 1,6 \\
\hline
\end{tabular}

Los vectores de evaluación global (columnas de promedios) señalan ya un primer indicador válido a través de sus promedios sobre las posibilidades relativas de los países A $(6,8)$ y $B(4,4)$.

El método se suele perfeccionar con la discusión (autorreflexibilidad sistémica) de cada una de las cifras de las matrices y la asignación sucesiva de probabilidades y expectativas matemáticas, de forma, por ejemplo, que al final se dispone de una sola cifra (entre 0 y 1) para cada país, lo que viene a significar la probabilidad estimada de que el negocio en cuestión tenga éxito. Un país por debajo del 0,5 sería radicalmente desestimado, mientras que uno que supere el 0,75 tiene grandes posibilidades de instalar la planta en su territorio. El método suele clasificar los países candidatos en función de estas condiciones objetivadas a través de un proceso interactivo e iterativo que reduce máximamente las posibilidades de error. M ayor es el número de criterios estimados, más alta la seguridad y fiabilidad del resultado.

Enfoque metodológico que igualmente podría aplicarse a cualquier tipo de empresa o acción, por ejemplo revolucionaria, donde un grupo de activistas, en lugar de utilizar la mera intuición o la siempre sospechosa autoridad de un líder, se propusiera objetivizar/consensuar al máximo la decisión definitiva. Los primeros criterios a evaluar entonces podrían ser el grado de vigilancia policial, de apoyo social, el día/hora óptimos, el espacio físico operativo, etc. Esta metodología tiene múltiples aplicaciones y no es raro que hayan sido precisamente científicos sociales los que han desarrollado y perfeccionado (0. H elmer, N. Rescher, B. Brown, N. D alkey, R. Campbell, y otros). En realidad, viene a suponer una aplicación práctica de lo que $\mathrm{H}$ abermas ha defendido como «intersubjetividad» en la conformación de lo teórico social.

Pretendo, pues, haber mostrado que es posible y deseable pasar del lenguaje común, impreciso y a menudo repleto de subjetividades espúreas, al len- 
guaje matemático/cuantitativo más exacto, criticable y apto para generar acuerdos intersubjetivos (Boudon, 1971). Aparte, claro está, de otras ventajas del lenguaje formalizado como las estenográficas o las deducibles. V éase además como gran parte de los problemas sociológicos de moda como la «reflexibilidad» (los expertos afinan sus cálculos a partir de las iteraciones), el «sujeto autodeterminado» (convertido al menos en actor autodeterminado para el problema concreto) o el «pensamiento de segundo orden» (por el proceso iterativo de asignación de grados), quedan en principio preresueltos, con lo que quiero decir, replanteados para mejorar sus posibles soluciones. El método D elfos al canza así, de una manera matemático-forzosa y consensuada, una solución sistemáticamente heterodeterminada superadora de la incertidumbre contingente inicial y pábulo además, por otra parte, para el planteamiento de nuevas incertidumbres de superior calado científico y sobre todo humanístico.

\section{3. ¿Sociología para quién?}

«Sociology for whom?» (M CC Lung Lee, 1906), or «k nowledge (sociology) for what?» (Lynd, 1939) son ya viejos avisos en pos de una ciencia social humanista, ciencia que en mi opinión debería comenzar formulándose la siguiente pregunta básica: «:Para qué el hecho social?». Sólo cuando respondamos a esta pregunta, quizás conozcamos el por qué último de la sociología y si en verdad nos estamos olvidando de algo. Resituemos, en una serie de puntos esquemá ticos, algunos de los posibles problemas relevantes del análisis sociológico.

a) Toda «sociedad» (desde la simple díada de individuos, al Estado-nación o las complejas sociedades supranacionales) busca y produce un cierto «valor añadido» que debe producirse por el mero hecho de la asociación. Si el sistema $\mathrm{S}$ formado por $\mathrm{n}$ individuos es capaz de «producir» más de lo que producirían los $n$ individuos actuando separadamente, entonces y solo entonces, nace la asociación, y por extensión cualquier tipo de sociedad (natural 0 artificial). Valor añadido que representa la esencia (origen y fin) de lo social y rasgo único que justifica su existencia.

b) Q ue, a pesar de la axialidad de este rasgo (repito, rasgo único que justifica la existencia de la relación asociativa), ninguna ciencia actual toma como objeto de análisis dicho valor añadido. Si los individuos nos asociamos, o simplemente colaboramos, es siempre para obtener un algo (material o inmaterial); sin embargo, dicho «algo» no es motivo de atención para los científicos sociales. Resulta por ello chocante que nos asociemos sólo para alcanzar unos fines, que ni antes como previsión, ni después como resultado, merecen nuestro análisis en tanto que tal val or añadido gl obal. Se presenta una situación tan absurda como aparentemente paradójica: H acemos $X$ con el fin exclusivo de alcanzar $Y$ para a continuación olvidarnos de $Y$ seguramente por estar enzarzados en el conocimiento de $X$. M as, ¿Cómo 
puede pretenderse el conocimiento de los factores que explican $Y$ si esta variable es desconocida? ¿C ómo podría calcularse, por ejemplo, en el modelo regresivo $Y=a_{1}, X+a_{2} X_{2}+\ldots a_{n} X_{n}$ la aportación de cada variable independiente $X$ (representada por los coeficientesa), si no conocemos cómo se comporta la variable dependiente Y? ¿C ómo podríamos explicar por qué un número tan bajo de mujeres ocupan un puesto relevante en la sociedad si no conocemos este número? ¿C ómo podemos explicar un fenómeno si no tenemos conciencia del mismo? D ado que metodológicamente el problema tiene una presentación absurda, las preguntas deben ir por otros derroteros. ¿Q ué factores mediáticos pueden estar impidiendo el análisis de los objetivos últimos de la actividad asociativa? ¿Cómo puede explicarse tan incongruente proceder? ¿Q ué ignotos intereses pueden estar obliterando (¿H asta cuándo?) el conocimiento de posiblemente lo único (sistema de valores) que interesa a la mayoría de los individuos?

c) Este apenas sostenible silencio sociológico sobre los fines de las unidades sociales, aparte los intereses que pueda representar (carácter parahomeostático de los sistemas sociales), se apoya en falacias que, como el teorema de imposibilidad de Arrow (uno de los más distorsionantes y regresivos que conozco), vienen a «demostrar» que no es posible la agregación de intereses individuales en una única función social de utilidad. H olgaría por tanto su construcción y cálculo y consagraría el velado desprecio (nunca asumido por supuesto) que se «merecen» las individuales opiniones, casi siempre «insuficientemente conocedoras» de la realidad o de sus propios intereses. La crítica sistemática al método de la encuesta estadística (quizás el instrumento más válido para agregar las preferencias de colectividades) frente a la defensa del método del grupo de discusión (epistemológicamente estéril para realizar tal agregación), contribuyen a mantener tan intrigante y ominoso silencio.

d) Q ue en la búsqueda de estos fines, los individuos nos organizamos como sistemas de transformación (Piaget, Easton, D eustch, Almond, Bunge) donde perseguimos convertir unos medios (recursos, materiales o no) en fines (sis tema de valores). La analogía gráfica Entradas (X) $\rightarrow$ Transformación (T) $\rightarrow$ Salidas (Y), representa con bastante exactitud lo que todo grupo real iza en cada acto de su existencia, sea o no (como monsieur J ourdan) consciente de ello.

e) Q ue dicha analogía nos permite conocer la eficiencia global de cual quier sociedad a través de la expresión $T=Y$ / X, esto es, T (la organización) será mayor cuanto mayores son las salidas $Y$ (o sistema de valores producido) y menores las entradas $X$ (o recursos utilizados), por ejemplo, el presente artículo representaría inevitablemente un sistema de tranformación donde utilizamos una serie de entradas o medios (principalmente nuestro tiempo) para obtener un sistema de valores (entre los que destaca sin 
duda el modesto conoci mi ento) que se derivaría de la evaluación crítica del lector. La analogía transformadora parece, pues, representar correctamente el proceso.

f) Lo que obtengamos ( $Y$ ) depende de $T$ (organización) y de $X$ (recursos), pero la experiencia ha demostrado que resulta mucho más decisiva o explicativa la T que la X. D e ahí la importancia creciente del concepto de organización basada en el conocimi ento de leyes y relaciones científicas entre los el ementos que componen los recursos utilizados (Bell, Tofler, N aisbitt, etc.). D e todo ello se puede establecer el siguiente silogismo:

1. Los individuos forman una asociación solo y exclusivamente porque persiguen un valor añadido ( $Y$ ), expresable solo y exclusi vamente éste por un sistema de valores.

2. Dicho valor añadido (sinergia social) es explicado, principalmente, por la propia organización ( $T$ ) interna a través de su capacidad de «autopoiesi s» sistémica ( $M$ aturana y Varela).

3. Luego el tema sociológico central no podría ser otro que el análisis (descripción y explicación) del conjunto T en tanto que sistema transformador, cosa que como sabemos no se hace, salvo, bastante imperfectamente por cierto, en el mundo empresarial, donde se ven obligados a considerar la empresa como un todo productor de beneficios o de pérdidas.

g) Q ue tanto Y (el sistema de valores producido), como X (los medios empleados), son en la mayoría de los casos válidamente cuantificables a través del conocido proceso de las definiciones operacionales (Lazarsfeld, 1971), que pasa de la imagen conceptual teórica al conjunto de indicadores empíricos (objetivos y subjetivos).

h) Q ueY puede, además, resultar comparable intersistémicamente, aún cuando se trate de sistemas social es culturalmente alejados o de dimensión y naturaleza heterogéneas. Posición naturalista que vengo suscribiendo desde hace tiempo y que considero heurísticamente justificable.

i) Q ue no parece finalmente lógico que la sociología consagrada al estudio de las unidades social es globales (estados-nación, instituciones, organizaciones, empresas, grupos, familias, etc., en suma, sistemas de individuos en interacción) no centre su atención (descriptiva, explicativa o predictiva) en el inevitable sistema de valores, que, como único fin, surge del no menos inevitable proceso entradas-transformación-salidas como conceptos operacionales, y con clara vocación y posibilidades de ser formalizadas (Cortés y otros, 1974) y cuantificados (Lazarsfeld). La sociología de estas unidades sociales se enriquecería así con interesantes análisis axiológicos de lo que se considera relevante para los individuos que componen la unidad 
social y considerablemente más científicos al emplear lenguajes formalizados. El objetivo social $(Y)$ sería una función de $(X)$ y viceversa, pero, como dije, no parecen posibles explicaciones adecuadas centradas solamente sobre los medios $(X)$ sin definiciones precisas de los fines sociales a conseguir $(Y)$. A la luz de estos considerandos cabe, pues, la duda de si la sociología no se olvida de lo más humanísticamente rel evante (los sistemas de valores perseguidos/al canzados por las unidades sociales), lo que podría fácilmente tratar de manera cuantificada y verificable, como cuando se aplica el balance socio-integrado de gestión (BASO IG) a la unidad empresarial.

\section{El objetivo olvidado: el «diferencial sinérgico»}

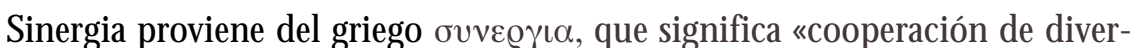
sas funciones dentro de un conjunto» (Lalande, 1968). Se aplica el término sinergia social para nombrar a la «productividad» añadida que obtienen determinadas personas por su mera colaboración. Percibir dicho diferencial exige determinar la siguiente desigualdad: EI sistema S compuesto de $n$ personas produce $Y$, mientras que las personas trabajando individualmente producen $p_{1}, p_{3}, \ldots p_{n}$, cuya suma total suele ser menor que lo producido por el sistema. Formalmente:

$$
Y>\sum_{y=1}^{n} p_{i}
$$

desigualdad fundamental que sirve para calcular lo que llamaré «D iferencial sinérgico» (DS) en la forma:

$$
D S=Y / \sum_{y=1}^{n} p_{i}
$$

donde si DS > 1 existe el «diferencial sinérgico positivo» que justifica el modelo societario, o esa especie de «plusvalía» generada por la colaboración social. El conocido ejemplo de la fabricación de alfileres de A. Smith (sinergia económica), o la mera supervivencia del recién nacido (sinergia vital) no son más que dos de entre la extensa tipología de sinergias posibles.

La unidad social (desde la pareja de enamorados a la más compleja organización) se convierte así en la célula fundamental de la sociedad moderna, trenzada por un sinfín de unidades sociales más o menos especializadas. Familias, escuelas, iglesias, empresas, burocracias, partidos, gobiernos, etc., son las unidades sociales de cuyos «diferenciales sinérgicos» dependemos de la cuna a la tumba, haciendo del mundo moderno algo inconcebible sin el funcionamiento, desarrollo, creación y ocaso de estas unidades.

Sin embargo, la ciencia parece haber dejado de lado el estudio de las unidades sociales como tales. Y si la sociología - ciencia de las agrupaciones humanas- no lo hace, ¿quién lo haría? Por mor de la división científico-académica, cada una de las especialidades que estudian lo social, terminan viendo y analizando su árbol correspondiente, pero ninguna de ellas percibe el bosque 
completo, esto es, el «diferencial sinérgico», precisamente lo que puede representar la contemplación más puramente sociológica de la unidad social, pues to que se presume como la única contemplación que interesa al Ilamado «hombre de la calle». M e refiero concretamente a tomar como objeto de investigación lo que puede llamarse «productividad añadida de una sociedad por el mero hecho de estar constituida como tal». Productividad que, en base a la desigualdad fundamental vista en [1] exige analizar como mínimo las tres siguientes fases:

1. La estructura de $Y$ en tanto que indica un complejo compuesto de dimensiones teóricas $D$ e indicadores empíricos I. Formalmente: $Y=f\left(D_{1}, D_{2}\right.$, $\ldots D n)$ donde $D_{i}=f\left(y_{1}, y_{2}, \ldots y_{n}\right)$. Toda unidad social debe, pues, conocer sus objetivos. Si no se conocen, ni se prevén, ni se comparan, el conocimiento de la unidad social resultará teóricamente deficiente o espurio y su gestión socialmente reprobable al olvidarse, analistas y gestores, de las necesidades y grados de satisfacción de las personas que la conforman.

2. El conocimiento de $X$ en tanto que función directa de los medios utilizados $m$. Formalmente: $X=f\left(m_{1}, m_{2}, \ldots m_{n}\right)$. Toda unidad social debe conocer los medios que utiliza. El coste total de los recursos utilizados para obtener los objetivos anteriores en tanto que medida del mas alto interés ecológico o negaentrópico.

3. El conocimiento final de $T$ en tanto que función de $Y$ y de $X$ en la expresión $T=Y$ / X, donde, en virtud de la estandarización previa de los indicadores y de su promedio final, si $T>1$ se produce una transformación positiva; si $\mathrm{T}<1$, negativa, y si $\mathrm{T}=1$, neutra.

El olvido de estas tres dimensiones resulta en una omisión ciertamente grave si tenemos en cuenta que ese bosque que se deja de percibir es un conjunto, no de árboles, sino de hombres y mujeres que sienten necesidades y que buscan satisfacerlas precisamente a través, y solo a través, de su interdependencia social «H uman Societies exist for only one reason: to unable humans to satisfy their needs» (Lenski \& Lenski, 1978: 33). 0 por decirlo con M ullins (1996: 294) cuando se refiere a las organizaciones: «strictly, organizations have no goals; only people do». El leitmotiv de toda sociedad, la satisfacción global - real y/o percibida - de su colectividad va a quedar así sin analizar. El conocido desenfoque de las especial idades científicas (nunca mejor recordar a 0 rtega y sus «bárbaros especialistas») Ileva a trocear la sociedad de manera tal que el resultado es parecido al análisis que puede hacerse de las piezas individualizadas de un motor desarmado. Su unidad, su funcionamiento, su finalidad, la razón de ser de cada una de sus piezas, queda ignorada.

Resulta así que los economistas estudian los medios de producción y se ocupan, en particular los modernos economistas, de unas pocas variables instrumental es como la inflación, los tipos de interés, la inversión, la balanza de pagos y al gunos otros. Todas ellas centradas al rededor de la oferta y la demanda, para, en definitiva, ocuparse de sólo uno de los valores perseguidos por la 
sociedad: el de riqueza material, lo que conlleva un notable sesgo en la evaluación de las variables y sus efectos sistémicos sobre la globalidad. El paro, por ejemplo, una importantísima variable social, es considerado por los economistas como un subproducto secundario que ha de someterse al imperio de las variables económicas citadas. C ontinuando con el especialismo, los antropólogos social es estudian las costumbres, los usos y folklores de aquellos pueblos y sociedades vinculados de forma más natural y menos artificiosa. Y aunque uno de los conceptos principales de la antropología social sea los valores, éstos suelen ser contemplados desde la diversidad (relativismo cultural), más que desde la confluencia de intereses personales en unos cuantos val ores centrales o valores-fines universales, a lo que habría que añadir las dificultades intrínsecas de la cuantificación socioantropológica. Por su parte, la psicología social estudia el comportamiento individual de la persona en sociedad y, aunque no olvida tomar al individuo como un conjunto de necesidades objetivas y subjetivas, se centra sobre todo en la serie de condicionantes sociales del comportamiento individual, pero no, naturalmente, en el comportamiento de la unidad social como ente global. La ecología humana se ocupa de la interacción y adaptación al medio físico de las sociedades tomando como sus conceptos centra les, el «nicho» o lugar que ocupan, la densidad, la dominación, la competitividad, la simbiosis biológico-fisica y otros. Los historiadores se ocupan de investigar el pasado centrando sus análisis, bien en el comportamiento de las grandes unidades sociopolíticas, bien en el estudio biográfico de personajes, pero no tanto en comparar qué sistemas de val ores-fines gl obal es han al canzado determinadas sociedades en el pasado y cuáles son las tendencias axiológicas de las mismas. La filosofía (lo que parece va quedando de ella) ha derivado principalmente hacia la lógica formal, según algunos, bastante alejada de las preocupaciones y problemas del mundo actual, lo que parece significar un cierto retroceso en relación con su problemática humanística clásica. M ención especial merece la ciencia política, quizás lo que más debería concretar y analizar las respuestas organizativo-sociales (políticas) a las demandas y necesidades de la población. D icha rama de la ciencia social cometería el error, dentro del actual currículum académico, de considerar al sistema político como un subsistema separado del sistema social al que trata como un entorno. Esta separación meramente académica y artificial, quizás montada sobre la base mimética del especialismo en boga, es lo que lleva a autores tan prestigiados como D avid Easton o G. Almond a considerar que las salidas del sistema político son «decisiones» y «acciones» para el primero y los tres conocidos poderes: legislativo, ejecutivo y judicial, para el segundo. N i una palabra (una excepción es D eustch) sobre las necesidades concretas de la colectividad y su respuesta política a las mismas, que deberían ser precisamente los objetivos centrales del análisis político-lógico. La ciencia política se suele aplicar así al estudio de los procesos electorales, el poder, los grupos de presión, las burocracias políticas, o las relaciones internacionales, pero apenas atacan el problema político axial que consiste en definir, medir y comparar lo que un sistema político debe hacer y hace en respuesta a las demandas concretas de la población, la cual, para más «inri» 
vota o escoge a los políticos sólo y exclusivamente para que realicen esta función olvidada. Por contra, una rama académica que si se ocupa de la «productividad global» de un sistema social, es la teoría de la organización empresarial. D esde los clási cos trabajos de Taylor y Fayol, pasando por los de D rucker, Perrow, Selznick Simon, Tannembaum, Argiris, Barnard, Etzioni, H erzberg, Likert y tantos otros, así como los esfuerzos de al gunas instituciones como el Center for Effective $O$ rganizations con sede en California, EE.U U ., o los interesantes trabajos recientemente publicados por la American Compensation Association (M cAdams \& H awk, 1994; M cAdams, 1996) van en este sentido, pero dichas tentativas adolecen de dos defectos notables: primero, no se plantean teóricamente la globalidad epistemológica y, segundo, no expresan las salidas del sistema en términos de valores-fines, únicos entes que pueden referenciarse directamente con las necesidades correspondientes de los individuos que componen su organización.

No parece existir, pues, una ciencia que se ocupe de explicar, medir y comparar los «beneficios» que dos personas obtienen por el hecho de colaborar entre ellas, 0 , en otras palabras, no existe una ciencia que se ocupe de definir, medir y analizar la «productividad global» 0 «valor añadido» que todo hecho societario implica. Productividad que forzosamente ha de expresarse en términos de val ores-fines, por ser precisamente aquello, y solamente aquello, lo que justifica la existencia de la propia sociedad. El tan perseguido diferencial sinérgi co queda decididamente olvidado. Extraño pero cierto.

A intentar llenar este hueco podría dirigirse una buena parte del trabajo sociológico actual, integrando la teoría básica de las necesidades humanas enmarcada en el todo social según los principios de la teoría de sistemas, especializada, como se sabe, en el tratamiento de los problemas y las unidades complejas. Se llamaría así enfoque axio-operacional al procedimiento de análisis social que combinase la utilización de la teoría de sistemas ya clásica (Von Bertalanfly, Buckley) y sus derivaciones cibernéticas (W iener, M aruyama), con las modernas aportaciones de la nueva teoría sociocibernética (Luhmann, Bailey, Geyer) y sobre todo con la parte de la axiología que estudia aquellos valores que se muestran comunes a la generalidad de los individuos (teoría objetivista de los val ores). Teoría, esta última, que obligaría a enfatizar el papel esencial de los valores en el análisis del «hecho societario» (H all, 1994).

Esta concepción axiológica del sistema social como ente compuesto por personas agrupadas hacia y por causa de la satisfacción de necesidades individuales, nos permite enunciar la razón de ser de todo sistema social en función ahora de su superior realización en términos de valores, que es lo que puede llamarse «emergencia axiológica de lo social», medible por la misma desigualdad pero donde los indicadores y son ahora valores (niveles) conseguidos. 0 sea, es sólo la superior capacidad para satisfacer carencias individuales a través de valores, Io que determina la existencia de los sistemas sociales.

Sucede a veces, sin embargo, que la desigual dad vista en [1] no se cumple en la dirección deseada, y por tanto $D S<1$, caso atípico que sólo se produce 
en determinados momentos de ruptura social. Lo normal, sin embargo, está representado por DS >1, justificando así la existencia de la sociedad en sus múltiples formas asociacionales. Luego Y es, o debería ser, salvo argumento en contra, el objeto privilegiado del sociólogo. Solo si dicha desigualdad es significativa se mantiene la sociedad, de lo contrario, se deshace la colaboración y ésta dejaría de existir, como suele suceder cuando se constata que Y ya no es lo suficientemente grande como para justificar la asociación, o cuando incluso se cumple lo contrario de lo previsto por la regla general, produciéndose la desigualdad negativa de la expresión. Las quiebras, las disoluciones, las separaciones o los divorcios son entonces el corolario lógico del fracaso social, Io que traduce la pequeñez 0 insuficiencia de $Y$ en relación con las expectativas inicialmente generadas.

\section{5. ¿Y la teoría sistémica?}

Pero $Y$, que representa un conjunto de beneficios (valores realizados) y que forzosamente ha de ser tratado como una unidad compleja o índice compuesto por dichos valores, no es analizada actualmente ni siquiera por la teoría de sistemas sociales, que además de social se supone que su papel central es el análisis de la globalidad. En dicha teoría de sistemas (en sus aplicaciones sociales), la cual ha presentado ya desarrollos importantes, $Y$ suele quedar ignorada. De esta forma la razón de ser del hecho social en tanto que sistema, la motivación profunda que da origen a las relaciones sociales, el cemento que mantiene unidos a los individuos, lo único y solo lo único, que justifica el hecho societario como sistema, jno es del interés ni siquiera de los sociólogos sistémicos! y así, en lugar de ocuparse del concepto Y (las salidas del sistema) se especializan en el tratamiento de los procesos sociales (Buckley), en las comunicaciones (Luhmann) de la entropía (Bailey), o de la «sociocibernética» (G eyer), curiosa pérdida de visión sistémica (del hecho social total, como diría M auss) con la cara pegada a cualquier árbol del bosque. $\mathrm{N}$ i siquiera el intento formalizador de C ortes, Przeworski y Sprague o el más reciente esfuerzo integrador de Bailey, ponen el necesario énfasis en las salidas. La nueva teoría de sistemas sociales, por lo tanto, habría de basarse en las notables aportaciones de Cortés y otros, Bailey, Luhmann y otros, pero superándolas a través de su integración con la teoría de los val ores.

La utilidad demostrada de las evaluaciones globales realizadas hasta ahora, tales como los sistemas de control de gestión en las empresas, los Balances sociales de las instituciones o el examen del «estado de la nación» en los debates parlamentarios, exigen un tipo de análisis/síntesis de la globalidad donde cualquier dimensión teórica ausente determinará irremisiblemente (epistemológicamente) la invalidez global del análisis por incompleto o parcial, cuando no sea tachado de veladamente manipulador.

¿Q ué requisitos ha de cumplir la nueva teoría sociosistémica que pretenda percibir la unidad de lo social? A parte los imperativos requisitos de «totalidad epistemología» y de «transformación», los siguientes: 
A. Sociologización. La sociedad ha de ser contemplada como interpretada por la totalidad de los individuos que la componen desde la perspectiva sociológica del «hombre de la calle», no meramente desde los despachos universitarios o científicos. El investigador social debe realizar el necesario esfuerzo de interpretación empática para ponerse en el lugar del ciudadano común y analizar cualquier organización social (grande o pequeña) desde lo que los individuos esperan de las mismas para satisfacer sus anhelos, intereses o carencias. La teoría sistémico-social no puede volver a cometer los desatinos que han cometido ilustres colegas que llegaron a definir nada menos que las sali das o realizaciones de los sistemas sociopolíticos como «decisiones» y «acciones» (Easton) o como «elaborar la regla», «ejecutar la regla «vigilarla judicialmente» (Almond) o como el célebre esquema AGIL (Parsons). ¿Podemos acaso criticar a los sistemas políticos utilizando estos conceptos? ¿Acaso no llevan a cabo, absolutamente todos los sistemas sin excepción, eficaces e ineficaces, gran profusión de decisiones, acciones, ela boraciones de reglas, etc. sin que por ello mejore el bienestar de la población? ¿Acaso puede evaluase o compararse una gestión, tanto de un sistema político como de una empresa o cualquier otra organización, en base a tan generales y meramente mediáticos conceptos? U na vez más, lo que el hombre de la calle espera de los sistemas políticos, son «beneficios» aplicables a sus necesidades como salarios, nivel de pensiones, buenas carreteras, seguridad ciudadana, libertades políticas, conservación de la natural eza, etc., serie de expectativas que llamaré valores-fines y que se estructurarían en un patrón referencial de valores (PRV) de aplicación general. A esta, a mi parecer ineludible, operación de interpretar las salidas de un sistema social desde la óptica carencial del hombre de la calle, es a lo que llamo «sociologización».

B. Axiologización. Los valores son tenidos como el reverso de la medalla de la necesidad (K luckhohn), y dado que el sistema social se forma sólo y únicamente para mejor satisfacer las necesidades individual es, las realizaciones o salidas de los sistemas sólo pueden lógicamente ser expresados en términos de valores. Para ello será preciso separar los valores en dos grandes categorías, a saber: I) los valores específicos y particulares a cada cultura o época (relativismo cultural) y 2) los valores generales o universales comunes a la práctica total idad de los individuos y pueblos al margen de cualquier contingencia espacio-temporal (teoría objetivista de los valores). En trabajos anteriores, he propuesto para su utilización, en cualquier tipo de sistema social, un patrón referencial de valores que se compone de los nueve siguientes val ores: salud, seguridad, conocimiento, libertad, justicia, riqueza material, conservación de la naturaleza, prestigio y calidad de las actividades. No referir, pues, las realizaciones (salidas) de los sistemas sociales a valores concretos que responden a necesidades concretas de personas concretas, podría suponer una mixtificación más o menos velada de la teoría social y un insospechado engaño a las personas que confor- 
man la sociedad y sostienen económicamente sus instituciones científicas y académicas.

C. Cibernetización. Si la cibernética es «la ciencia de la comunicación y el control» (W iener), o «la ciencia que rinde eficaz la acción» (C ouffignal), por cibernetización del sistema hemos de entender la vigilancia que los interesados han de ejercer para que las salidas o los estados finales del sistema se atengan a lo previamente especificado o proyectado. Afortunadamente la teoría de la empresa ha desarrollado procedimientos de control de gestión (previsión/ejecución/desviación) aptos para ser generalizados al resto de los sistemas sociales. La sociocibernética se presenta hoy, pues, como una de las ramas de la teoría de sistemas sociales con más posibilidades de aplicación. Es lo menos que puede esperarse de los responsables de cualquier gestión: vigilar las realizaciones para corregirlas a tiempo si procede.

D . Autocrítica. La cibernetización del sistema permite precisamente la autocrítica de su comportamiento, no sólo por el típico análisis de las desviaciones que permite el control de gestión, sino también porque la totalidad de los valores y de los indicadores que los representen, pueden quedar expresados dentro de un intervalo común o estándar que variará de 0 (mínimo, pésimo) hasta 100 (máximo, óptimo), y serán las diferencias entre lo conseguido y lo óptimo (o lo proyectado) lo que conllevará indefectiblemente una crítica radical y comparable de lo que el sistema ha podido hacer y, sin embargo, no ha hecho en cada uno de los objetivos o indicadores. A la teoría sistémico-social más moderna (la que surge de los últimos desarrollos sobre los conceptos de autopoiesis, reflexibilidad, entropía, cibernética de segundo orden, tercera cibernética y otros, que se complementarían con la exigencia de los cuatro requisitos citados, es a lo que llamo enfoque axio-operacional, método que no hace sino intentar interpretar rigurosamente el principio de complejidad de los sistemas sociales y sus derivados de parsimonia y relevancia para separar lo importante de lo secundario, lo esencial de lo que no lo es, lo que busca y lo que no busca el hombre de la calle.

Frente a la descarnada visión que del sistema social tienen autores como Luhmann, para quien la persona humana desaparece prácticamente del sistema, véase, por ejemplo, la idea que sostiene la Conferencia de las $\mathrm{N}$ aciones U nidas sobre salud y ambiente en el desarrollo humano sostenible: «Se entiende que el desarrollo humano sostenible es un proceso que mejora la suerte de los seres humanos - un proceso que es holístico, integrado eintegrador de los elementos que conforman la totalidad ambiental- ; un proceso en el cual los elementos y las partes sólo pueden ser evaluados significativamente en su relación con el todo. Los humanos son tanto los actores como los beneficiarios del proceso y su supervivencia y bienestar son su razón de ser» (Jiménez H errero, 1997: 72). 


\section{6. ¿Una hipótesis plausible?}

Podría no existir contradicción ni paradoja alguna en el olvido de Y por parte de los científicos sociales. Al contrario, resulta lógico que el poder (vía financiación y otros medios) procure velar la parte considerable mayor de Y que percibe, para que no quede constancia (verificable) del hecho. Conocido es el recelo del poder hacia toda transparencia contable. (En el mundo empresarial, por ejemplo, coexisten a veces todavía hasta tres contabilidades: una para el consejo de administración, otra para el fisco y otra para negociar los convenios colectivos.) En los términos exclusivos del valor añadido Y, entendido «como suma cero» (lo que yo tomo tu no), el poder detesta el número; o mejor dicho, detesta la publicidad del número, aunque se regodee, sin duda, en su contemplación privada. D e ahí que termine por institucionalizar las cuentas bancarias numeradas (secretas) o la no publicación por parte de los organismos públicos de sus declaraciones (¿sistemáticamente fal sas?) de sus rentas. Si hay algo que el poder tema y evite es que se cuantifique lo que realmente toma del añadido sinérgico producido por la relación social. La vieja plusvalía marxista no le suena precisamente a tranquilizante canción de cuna. Esperpéntica presentación de la realidad social en la que no dejan de participar, conscientes o no, los propios científicos sociales.

Los medios para conseguir el «silencio cuantitativo» sobre $Y$ por parte de éstos últimos, son tan variados como sutiles. Comienzan por aprovechar la natural complejidad de Y para matrimoniarla precipitadamente con la hiperespecial ización científica y el desguace o despiece del objeto (la unidad social) al estilo de las Ilamadas «ciencias duras», pensando quizás que así el trabajo presentado es más científico. Todo ello en un ejercicio de mimetismo acomplejado merecedor de tan acerada crítica como de freudiana interpretación. No importa que a los políticos, a los padres de familia, a los directores de escue la, a los rectores de universidad, a los presidentes de clubes deportivos, eincluso a los empresarios más evolucionados y conscientes, y en general a todos los responsables de unidades sociales en funcionamiento, sólo les preocupe el contenido Y y vivan y trabajen para conocer y mejorar esta variable. Los científicos sociales no se inmutan por ello. Al contrario, al dar vueltas al rededor de los microobjetos que ellos mismos arrancan de la unidad, despojándolos por tanto de sus determinantes estructurales, logran matar dos o tres pájaros de un tiro: «salvan» la complejidad de Y; utilizan el método científico por creerse equipados con dudosos «microscopios» sociales, y de paso dejan intocados ciertos intereses proclives a financiar dichas «investigaciones». La reciente historia de los trabaj os sobre el paro en España demuestra esta desagregación artificial, lo que está llevando al fracaso comparativo de su solución.

El Ilamado «pensamiento posmodernista», explicable fruto de la malintegración de una serie concomitante de circunstancias históricas (por ejemplo influencia de los relativistas Einstein, H eisemberg, G odel... ; del fracaso de la U RSS y su concepto político del todo; la complejidad del nuevo mundo y la sensación de impotencia ante sus problemas, etc.), al parecer convertido en 
una especie de fundamentalismo filosófico como única terapia contra el aburrimiento o la esterilidad, viene renunciando a cualquier intento de validación científica de los hechos: «M ás que ningún otro, este fin de siglo al que nos estamos aproximando se caracteriza por la concepción, difundida en todos los ámbitos científicos, de que no hay una racionalidad definitivamente válida 0 legitimable, sino sólo "paradigmas" locales, válidos en un sentido limitado» (Fisher, 1997: 12). 0, como más escuetamente lo expresó N ietzsche, uno de los padres de este movimiento: «N o hay hechos, sino únicamente interpretaciones» (Fisher, 1997: 19). Si la sonrisa de N ewton pudo ser leve y condescendiente, las carcajadas de los poderosos debieron oírse en $\mathrm{M}$ arte.

Y si así comienzan, prosiguen aprovechándose de la escasa sonoridad de las cifras (iqué aburrimiento!) frente a la combinatoria poética de las palabras, a los que, prestos, añaden la alergia que produce la inadecuada enseñanza de las matemáticas y su correspondiente retahíla de angustias y suspensos académicos. El número, tenido por clarificador y ordenador de la mente, termina enturbiándola. El «tiro al blanco» al símbolo matemático es entonces aprovechado por el poder para explotarlo en las ciencias sociales más molestas o peligrosamente críticas (aquellas, por ejemplo, que se ocupan cuantitativamente del reparto de los sistemas de val ores producidos), negando además (y certificándolo) cualquier posibilidad de cuantificar valores y preferencias, sustracciones y provechos. La numerofobia sobre los hechos relevantes se instala de tal forma en la sociología, que terminan siendo mayoría (a pesar de la inicial física social de Q uetelet y C omte) los oficiantes de las versiones literaria o hermenéutica, quizás una de las causas de la escasa madurez de la sociología como ciencia (no por joven), y su marginación creciente en los ámbitos universitarios (véase la tendencia de EE.UU .; y en España, lo sucedido en las facultades de económicas, por no mencionar la crisis de la filosofía, antecedente de lo que puede esperarle a la sociología «filosófica»). Pero la sutil penetración antinumérica alcanza cotas preocupantes cuando cabezas tan alertadas como las de nuestros $C$. M oya o J. I báñez, por no hablar del caso Cicourel, terminan donde empezaron: sin llegar a hacer unas cuentas que podrían haber resultado dramáticamente clarificadoras e intersubjetivamente verificables del posible abuso de poder cometido por la punta del iceberg sobre el resto, y como ellos mismos dicen, cuando critican los números «en lugar de las cuentas se presentan cuentos». La faena elitista queda primorosamente redondeada cuando se asume por la tribu sociológica que la emisión de predicados verbales no falseables significa admirativamente «parir palabras», mientras que la emisión de predicados cuantitativo-falsables significa despectivamente «cagar números».

En resumen, añadiendo las comprensibles actitudes elitistas a la feble estética del número y su temida y desafortunada enseñanza, explicaríamos en gran parte la numerofobia más típica de la sociología, produciéndose entonces, por parte de escribanos y orates, una lamentable descuantificación de lo relevante y una generalizada amnesia del objeto motivador de lo social. Y en lugar de integrar la palabra con el número, lograríamos a la vez anatematizar el núme ro e invalidar (científicamente) la palabra. Todo vale y nada vale. 
Aunque he de reconocer que, después de treinta años de oficio, ya no sé muy bien si es la sociología quien se olvida de su objeto o soy yo quien no ve ni la sociología.

\section{Bibliografía}

Boudon, R. (1971). La Crise de la Sociologie. Questions d'épi stemologie sociologique. Ginebra: Librairie D roz.

Cortés, Fernando; Przew orski, Adam; Sprague, John (1974). Systems Analysis for Social Scientists. N ueva York: A W iley Interscience Publication H ohn W iley \& Sons.

Fischer, H.R.; Retzer, A.; Schwizer, J. (1997). El final de los grandes proyectos. Barcelona: G edisa.

JACOBY, Russell (1977). La amnesia social. Barcelona: Bosch.

Jiménez H ERRERO, Luis (1997). D esarrollo Sostenible y Economía Ecológica. M adrid: Síntesis.

H ALL, Brian P. (1994). Values Shift a guide to personal \& organizational transformation. USA. Twin Lights Publishers, Inc.

LACH EN M EYER, Charles (1971). The language of Sociology. N ueva York y Londres: Columbia U niversity Press,

LALAN DE, André (1968). Vocabulaire Technique et Critique de la Philosophie. París: Presses U níversitaires de France.

LAZARSFELD, P. (1971). «M éthodes de la sociologie, le vocabulaire des sciences sociales». N etherlands: M ounton $\& \mathrm{C}^{\circ}$ and $\mathrm{M}$ aison des Sciences del ' $\mathrm{H}$ omme.

LEM ERT, Charles C. (1979). «Sociology and the twilight of man, H omacentrism and discourse in sociological theory». Londres y Amsterdam: Southern Illinois U niversity Press $C$ arbonadale Feffer $\&$ Simons, Inc.

LYN D, R.S. (1939). Knowledge for W hat? Princenton University Press.

LEN SKI, G erard; LEN SKI, Jean (1978). H uman Soci eties an introduction to macrosociology. USA. M CGraw-Hill Book C .

M CADAM S, Jerry L. (1996). The Reward Plan Advantage a manager's guide to improving business performance through people. San Francisco: Jossey Bass Publishers.

M CADAM S, Jerry L.; H AWK, Elizabeth J. (1994). «O rganizational Performance $\&$ Rewards 663 experiences in making the link». A research projecto of ACA.

M CC LUN G LeE, Alfred (1906). Sociology for Whom? N ueva York: O xford University Press.

M ULLINS, Laurie J. (1996). M anagement and Organisational Behaviour. Londres: Pitman Publishing.

SCH WARTZ, H oward; J ACO BS, Jerry (1979). Q ualitative Sociology a method to the madness. N ueva York. The Free Press. 